\title{
Three new species of Mesacanthion Filipjev, 1927 (Nematoda: Thoracostomopsidae) from Argentine coasts
}

\author{
Virginia LO RUSSO ${ }^{\circledR 1, *} \&$ Catalina T. PASTOR DE WARD ${ }^{2}$ \\ ${ }^{1,2}$ Laboratorio de Meiofauna Marina (LAMEIMA) - Instituto de Diversidad y Evolución Austral \\ (IDEAus-CONICET), Blvd. Alte. Brown 2915, U9120ACF Puerto Madryn, Chubut, Argentina. \\ "Corresponding author: lorusso@cenpat-conicet.gob.ar \\ ${ }^{2}$ Email: pastor@cenpat-conicet.gob.ar \\ ${ }^{1}$ urn:1sid:zoobank.org:author:A67891F9-E1BD-407D-AA67-E2ED5C722A21 \\ ${ }^{2}$ urn:lsid:zoobank.org:author:421DA5CB-6FDD-4219-BC2C-79AE72A2E8F1
}

\begin{abstract}
Three new species of Mesacanthion Filipjev, 1927 were found along Patagonian coasts (Argentina). Mesacanthion bifidum sp. nov. is characterized by short labial and cephalic setae, onchia of equal size, spicule arcuate, and gubernaculum with caudal apophysis, ending in two teeth. The species is related to M. virile (Ditlevsen, 1930) De Coninck \& Schuurmans Stekhoven, 1933. However, the spicules and gubernaculum of both species are different in shape. Mesacanthion longigubernaculum sp. nov. is characterized by its long and slender body, striated cuticle, relatively long cephalic and cervical setae, onchia of different sizes, amphidial fovea lentil-shaped, spicule arcuate, gubernaculum surrounding the spicule, and tail conical-cylindrical with terminal setae. Mesacanthion sanantoniensis sp. nov. is characterized by its long and stout body, striated cuticle, long cephalic setae, onchia of different sizes, amphidial fovea pouch-shaped, spicule arcuate, gubernaculum with dorsal apophysis, and tail conical without terminal setae. Following the key of Jeong et al. (2019), the last two species are related to M. pali Wieser, 1959 and M. longissimesetosum Wieser, 1953, so we provide a key to differentiate the four species.
\end{abstract}

Keywords. Free-living marine nematodes, diversity, taxonomy, Patagonia, coastal area.

Lo Russo V. \& Pastor de Ward C.T. 2021. Three new species of Mesacanthion Filipjev, 1927 (Nematoda: Thoracostomopsidae) from Argentine coasts. European Journal of Taxonomy 787: 17-31. https://doi.org/10.5852/ejt.2021.787.1611

\section{Introduction}

Since the middle of the 1970s, the free-living marine nematodes have been studied uninterruptedly in the Argentine coasts, the southernmost continental region of South America. More than 400 species have been distinguished along the coastal area that extends over $4725 \mathrm{~km}$. The purpose of the studies was not only for ecological investigations but taxonomical. On the other hand, as most of the species found were new to science, intense work has been carried out over the years to try to describe them. To 
date, descriptions of 74 species have been published. Among the species, some belonging to the genus Mesacanthion Filipjev, 1927 were found.

The family ThoracostomopsidaeFilipjev, 1927 consists of three subfamilies: ThoracostomopsinaeFilipjev, 1927, Trileptiinae Gerlach \& Riemann, 1974 and Enoplolaiminae De Coninck, 1965. Enoplolaiminae can be distinguished from the other subfamilies by their buccal cavity with three mandibles and three teeth. It has 17 genera (excluding Hyptiolaimus Cobb, 1930). Six species of Enoplolaiminae have been described in Argentina as new to science. They belong to the genera Enoplolaimus De Man, 1893 (1), Epacanthion Wieser, 1953 (2), Mesacanthoides Wieser, 1953 (1) and Parasaveljevia Wieser, 1953 (2).

Mesacanthion belongs to Enoplolaiminae. The most recent revision of Mesacanthion was from Jeong et al. (2019). An emended diagnosis is given as well as a list of valid species and species inquirenda and nomina nuda, a table with a comparison of diagnostic morphological characters of all species and a tabular and pictorial key to species with spicules shorter than two anal body diameters. After Jeong et al. (2019) the genus Mesacanthion has 39 valid species, 38 from marine environments and one from a freshwater environment. Three species were originally described in South America: Mesacanthion longissimesetosum Wieser, 1953 (Chile); M. proximum Gerlach, 1957 (Brazil); and M. rigens Gerlach, 1957 (Brazil). Another five species have been recorded for the region: M. hirsutum Gerlach, 1953; M. infantile (Ditlevsen, 1930) De Coninck \& Schuurmans Stekhoven, 1933; M. longispiculum Gerlach, 1954; M. majus (Filipjev, 1927) Gerlach \& Riemann, 1974; and M. virile (Ditlevsen, 1930) De Coninck \& Schuurmans Stekhoven, 1933. In the present work, we describe the first three new species of Mesacanthion for the Argentine coast.

\section{Material and methods}

\section{Description of the study sites}

Samples were collected from three different areas along the Patagonian Atlantic coast, Argentina (Fig. 1). The northernmost site, the city of San Antonio Oeste (40 $\left.43^{\prime} \mathrm{S}, 64^{\circ} 58^{\prime} \mathrm{W}\right)$, is in San Antonio Bay, located in the northwest part of San Matías Gulf. About 220 kilometres to the south are two other sites, Puerto Madryn city $\left(42^{\circ} 45^{\prime} \mathrm{S}, 65^{\circ} 02^{\prime} \mathrm{W}\right)$ and Bahía Kaiser ( $\left.42^{\circ} 46^{\prime} \mathrm{S}, 64^{\circ} 59^{\prime} \mathrm{W}\right)$, both in Nuevo Gulf. The southernmost site, Rada Tilly ( $\left.45^{\circ} 55^{\prime} 07^{\prime \prime} \mathrm{S}, 67^{\circ} 32^{\prime} 79^{\prime \prime} \mathrm{W}\right)$, is located in the middle part of San Jorge Gulf.

\section{Sample collection and treatment}

Samples were collected using a cylindrical plexiglass corer, fixed in 5\% formaldehyde prepared in filtered seawater with rose Bengal, and then the fixed nematodes were sieved through both $500 \mu \mathrm{m}$ and $50 \mu \mathrm{m}$ mesh sieves. The nematodes retained on the $50 \mu \mathrm{m}$ mesh were separated from the sediment by Ludox ${ }^{\circledR}$ TM and transferred to pure glycerin through a solution of ethanol: water: glycerin in 2:2:1 proportions and left at least one week in a desiccator. After that, they were mounted on glass slides sealed with CANADAX resin.

\section{Specimen analysis}

Morphometric data were obtained from camera lucida drawings using a Zeiss Standard WL microscope (D-7082 Oberkochen) with differential interference contrast (DIC). The measurements are given in micrometers. Images were taken using a Leica DM2500 microscope with incorporated camera. The literature was obtained from NeMys (Bezerra et al. 2021). The De Man's ratios 'a', 'b' and 'c' used in this paper were calculated as usual.

\section{Institutional abbreviations}

CNP-NEM = Collection of Nematodes of the Patagonia National Center (Centro Nacional Patagónico) 
MACN-In = Invertebrate Collection of the Argentine Museum of Natural Science 'Bernardino Rivadavia' (Museo Argentino de Ciencias Naturales 'Bernardino Rivadavia')

\section{Abbreviations for morphological terms}

$\mathrm{a} \quad=$ body length/maximum body diameter

abd $=$ anal body diameter

$\mathrm{b}=$ body length/pharynx length

$\mathrm{c}=$ body length/ tail length

$c^{\prime}=$ tail length/body width at level of cloacal opening or anus

cbd $=$ corresponding body diameter

$\mathrm{L}=$ total body length

\section{Results}

Phylum Nematoda Potts, 1932

Class Enoplea Inglis, 1983

Order Enoplida Filipjev, 1929

Suborder Enoplina Chitwood \& Chitwood, 1937

Superfamily Enoploidea Dujardin, 1845

Family Thoracostomopsidae Filipjev, 1927

Subfamily Enoplolaiminae De Coninck, 1965

Genus Mesacanthion Filipjev, 1927

\section{Type species}

Mesacanthion lucifer (Filipjev, 1927) Gerlach \& Riemann, 1974

Diagnosis (after Jeong et al. 2019)

Outer labial and cephalic setae situated at middle or anterior end of cephalic capsule. Mandibles welldeveloped, provided with claws, arch-shaped, each consisting of two rod-like columns anteriorly united by a curved bar. Teeth shorter than mandibles. Spicule mostly short, unipartite and symmetrical, sometimes long, bipartite (divided by a seam: M. ditlevseni (Filipjev, 1927) Gerlach \& Riemann, 1974) and asymmetrical (anisomorphic and anisometric: M. diplechma (Southern, 1914) Filipjev, 1927). If long, usually gubernaculum present with caudal apophysis. Marine and freshwater.

Mesacanthion bifidum sp. nov.

urn:lsid:zoobank.org:act:62E01288-0803-428A-BB5E-D51B592D1591

Figs 2, 5D

\section{Etymology}

In reference to the proximal part of the gubernaculum that ends in two small pointed teeth, from the Latin word 'bifidum'.

\section{Material examined}

\section{Holotype}

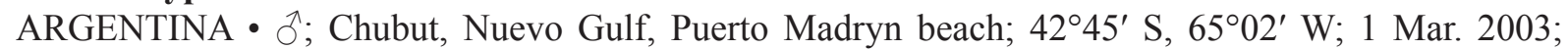

C. Pastor and V. Lo Russo leg.; high tide; fine sand sediments; CNP-NEM 936. 
Table 1 (continued on next page). Measurements ( $\mu \mathrm{m})$ of Mesacanthion bifidum sp. nov., M. longigubernaculum sp. nov. and M. sanantoniensis sp. nov., including mean values (range).

\begin{tabular}{|c|c|c|c|c|c|c|}
\hline \multirow[b]{2}{*}{ Character } & \multicolumn{2}{|c|}{$\begin{array}{l}\text { M. bifidum } \\
\text { sp. nov. }\end{array}$} & \multicolumn{2}{|c|}{$\begin{array}{l}\text { M. longigubernaculum } \\
\text { sp. nov. }\end{array}$} & \multicolumn{2}{|c|}{$\begin{array}{l}\text { M. sanantoniensis } \\
\text { sp. nov. }\end{array}$} \\
\hline & Holotype $\sigma^{\wedge}$ & 우 & Holotype $\sigma^{\wedge}$ & $\hat{0} \hat{0}$ & Holotype $\sigma^{\wedge}$ & 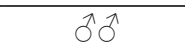 \\
\hline Number of specimens & 1 & 3 & 1 & 2 & 1 & 2 \\
\hline $\mathrm{L}$ & 2990 & $\begin{array}{c}3363.3 \\
(3150-3515)\end{array}$ & 1450 & $\begin{array}{c}1445.7 \\
(1380-1507)\end{array}$ & 2540 & $\begin{array}{c}2466.7 \\
(2120-2740)\end{array}$ \\
\hline a & 19.9 & $\begin{array}{c}25 \\
(22.8-26.2)\end{array}$ & 55.8 & $\begin{array}{c}52.9 \\
(47.8-55.8)\end{array}$ & 20.8 & $\begin{array}{c}24.7 \\
(20.8-29.4)\end{array}$ \\
\hline $\mathrm{b}$ & 4.9 & $4.9(4.6-5.2)$ & 4.1 & $\begin{array}{c}4.5 \\
(4.1-5.4)\end{array}$ & 4.2 & $\begin{array}{c}4.1 \\
(3.7-4.3)\end{array}$ \\
\hline c & 13.8 & $\begin{array}{c}14.7 \\
(13.8-15.7)\end{array}$ & 13.9 & $\begin{array}{c}14.6 \\
(13.9-15.1)\end{array}$ & 12.3 & $\begin{array}{c}13.1 \\
(12.3-13.9)\end{array}$ \\
\hline Inner labial setae length & 7 & $\begin{array}{c}8 \\
(7-9)\end{array}$ & 8.5 & $\begin{array}{c}8 \\
(7.5-8.5)\end{array}$ & 7.5 & $\begin{array}{c}7.3 \\
(7-7.5)\end{array}$ \\
\hline Outer labial setae length & 23 & $\begin{array}{c}20.5 \\
(20-21)\end{array}$ & 18 & $\begin{array}{c}19 \\
(16-23)\end{array}$ & 36 & $\begin{array}{c}33 \\
(28-36)\end{array}$ \\
\hline Cephalic setae length & 18 & $\begin{array}{c}13 \\
(12-14)\end{array}$ & 13 & $\begin{array}{c}12 \\
(9-14)\end{array}$ & 21 & $\begin{array}{c}20.2 \\
(17.5-22)\end{array}$ \\
\hline Buccal cavity width & 13 & $\begin{array}{c}22.3 \\
(18-27)\end{array}$ & 8 & $\begin{array}{c}10.3 \\
(8-13)\end{array}$ & 16 & $\begin{array}{c}15 \\
(14-16)\end{array}$ \\
\hline Buccal cavity length & 18 & $\begin{array}{c}19.3 \\
(17-21)\end{array}$ & 12 & $\begin{array}{c}10.7 \\
(10-12)\end{array}$ & 16 & $\begin{array}{c}16.7 \\
(16-17)\end{array}$ \\
\hline $\begin{array}{l}\text { Cephalic diameter at cephalic setae } \\
\text { level }\end{array}$ & 29 & $\begin{array}{c}39.3 \\
(38-41)\end{array}$ & 18 & $\begin{array}{c}18.8 \\
(18-20)\end{array}$ & 34 & $\begin{array}{c}31.3 \\
(29-34)\end{array}$ \\
\hline Amphid aperture width & 2 & $\begin{array}{c}1.9 \\
(1.8-2)\end{array}$ & 3 & $\begin{array}{c}3.5 \\
(3-4)\end{array}$ & 2 & $1.8(1.5-2)$ \\
\hline Body diameter at amphid level & 36 & $\begin{array}{c}50.7 \\
(48-52)\end{array}$ & 21 & $\begin{array}{c}21 \\
(21-21)\end{array}$ & 38 & $\begin{array}{c}36.3 \\
(32-39)\end{array}$ \\
\hline Amphid width/cbd (\%) & 5.6 & $\begin{array}{c}3.8 \\
(3.5-4.2)\end{array}$ & 14.3 & $\begin{array}{c}16.7 \\
(14.3-19)\end{array}$ & 5.3 & $\begin{array}{c}5.1 \\
(3.8-6.3)\end{array}$ \\
\hline Cephalic capsule length & 29 & $\begin{array}{c}34 \\
(32-36)\end{array}$ & 17 & $\begin{array}{c}16 \\
(14-17)\end{array}$ & 29 & $\begin{array}{c}27.8 \\
(26.5-29)\end{array}$ \\
\hline Cervical setae & 10 & $\begin{array}{c}15 \\
(14-16)\end{array}$ & $9.5 / 20$ & $\begin{array}{c}13.3 \\
(6.5-20)\end{array}$ & $9 / 13$ & $\begin{array}{l}10.2(7.5- \\
14)\end{array}$ \\
\hline Nerve ring from anterior end & 205 & $\begin{array}{c}241.7 \\
(210-265)\end{array}$ & 125 & $\begin{array}{c}110 \\
(92-125)\end{array}$ & 210 & $\begin{array}{c}200 \\
(170-220)\end{array}$ \\
\hline Body diameter at nerve ring level & 70 & $\begin{array}{c}89.7 \\
(87-94)\end{array}$ & 25 & $\begin{array}{c}26.7 \\
(24-31)\end{array}$ & 76 & $67(48-77)$ \\
\hline Pharynx length & 615 & $\begin{array}{c}686.7 \\
(680-700)\end{array}$ & 350 & $\begin{array}{c}325.3 \\
(256-370)\end{array}$ & 600 & $\begin{array}{c}603.3 \\
(570-640)\end{array}$ \\
\hline $\begin{array}{l}\text { Body diameter at pharynx base } \\
\text { level }\end{array}$ & 96 & $\begin{array}{c}121.7 \\
(115-132)\end{array}$ & 24 & $\begin{array}{c}25.7 \\
(23-30)\end{array}$ & 102 & $\begin{array}{c}91.3 \\
(60-112)\end{array}$ \\
\hline Maximum body diameter & 150 & $\begin{array}{c}134.7 \\
(132-138)\end{array}$ & 26 & $\begin{array}{c}27.5 \\
(25-31.5)\end{array}$ & 122 & $\begin{array}{c}103(72- \\
122)\end{array}$ \\
\hline $\begin{array}{l}\text { Anal/cloacal distance from anterior } \\
\text { end }\end{array}$ & 2774 & $\begin{array}{c}3133.7 \\
(2922-3291)\end{array}$ & 1345.5 & $\begin{array}{c}1346.5 \\
(1287-1407)\end{array}$ & 2334 & $\begin{array}{c}2277 \\
(1967-2530)\end{array}$ \\
\hline Anal/cloacal body diameter & 63 & $\begin{array}{c}59.7 \\
(57-63)\end{array}$ & 22.5 & $\begin{array}{c}22.5 \\
(20-25)\end{array}$ & 55 & $46.7(36-55)$ \\
\hline Spicule length as arc & 93 & - & 22 & $\begin{array}{c}22.7 \\
(22-23)\end{array}$ & 71.5 & $\begin{array}{c}71.2 \\
(69-73)\end{array}$ \\
\hline Spicule length as cloacal diameter & 1.5 & - & 1 & $\begin{array}{c}1 \\
(0.9-1.1)\end{array}$ & 1.3 & $1.6(1.3-1.9)$ \\
\hline Gubernaculum length & 24 & - & 14 & $\begin{array}{c}13.7 \\
(10-17)\end{array}$ & 33 & $\begin{array}{c}30.3 \\
(27-33)\end{array}$ \\
\hline Gubernaculum apophysis length & 21 & - & - & - & - & - \\
\hline
\end{tabular}


Table 1 (continued).

\begin{tabular}{|c|c|c|c|c|c|c|}
\hline \multirow[b]{2}{*}{ Character } & \multicolumn{2}{|c|}{$\begin{array}{l}\text { M. bifidum } \\
\text { sp. nov. }\end{array}$} & \multicolumn{2}{|c|}{$\begin{array}{l}\text { M. longigubernaculum } \\
\text { sp. nov. }\end{array}$} & \multicolumn{2}{|c|}{$\begin{array}{l}\text { M. sanantoniensis } \\
\text { sp. nov. }\end{array}$} \\
\hline & Holotype $\sigma^{\lambda}$ & 우우 & Holotype ${ }^{\hat{}}$ & $\hat{\partial} \hat{0}$ & Holotype $\sigma^{\lambda}$ & $\hat{\partial} \hat{0}$ \\
\hline Gubernaculum as spicule length (\%) & 25.8 & - & 63.6 & $\begin{array}{c}60.3 \\
(43.5-73.9)\end{array}$ & 60 & $\begin{array}{c}59.1 \\
(42.5-75)\end{array}$ \\
\hline Precloacal organ length & 34 & - & 5 & $\begin{array}{l}4.7 \\
(4-5)\end{array}$ & 21 & $\begin{array}{c}19.3 \\
(17-21)\end{array}$ \\
\hline Precloacal organ from anterior end & 2520 & - & 1290 & $\begin{array}{c}1317 \\
(1233-1428)\end{array}$ & 2310 & $\begin{array}{c}2163.3 \\
(1790-2390)\end{array}$ \\
\hline $\begin{array}{l}\text { Vulva from anterior end/Body length } \\
(\%)\end{array}$ & - & $\begin{array}{c}51.5 \\
(51.1-52)\end{array}$ & - & - & - & - \\
\hline Tail length & 216 & $\begin{array}{c}229.7 \\
(224-237)\end{array}$ & 104.5 & $\begin{array}{c}99.2 \\
(93-104.5)\end{array}$ & 206 & $\begin{array}{c}189.7 \\
(153-210)\end{array}$ \\
\hline$c^{\prime}$ & 3.4 & $\begin{array}{c}3.9 \\
(3.6-4.2)\end{array}$ & 4.6 & $\begin{array}{c}4.4 \\
(4-4.7)\end{array}$ & 3.7 & $\begin{array}{c}4.1 \\
(3.7-4.3)\end{array}$ \\
\hline
\end{tabular}

Paratypes

ARGENTINA 11 ; ; same collection data as for holotype; CNP-NEM $937 \cdot 2$ 우; Chubut, Nuevo Gulf, Bahía Kaiser; 4246’ S, 6459’ W; 8 m b.s.l.; Oct. 1997; C. Pastor leg.; CNP-NEM 938 to 939.

\section{Description}

\section{Measurements}

See Table 1.

Male (holotype)

Large and stout body. Cuticle smooth. Few short (about 9-12 $\mu \mathrm{m}$ long) somatic setae scattered along body. Cephalic region set-off with presence of cephalic capsule (17 $\mu \mathrm{m}$ in height). Cephalic capsule with same thickness throughout its rough surface. Anterior edge located at level of cephalic setae. Posterior end with no real incisions forming lobes but with slightly scalloped edge. Three rounded lips. Each lip carries two inner slender labial setae $(9 \mu \mathrm{m}$ long). Six outer labial setae $(18 \mu \mathrm{m}$ long) and four barely shorter cephalic setae (17 $\mu \mathrm{m}$ long) located at anterior of cephalic capsule, arranged in single crown. Immediately posterior to cephalic capsule four short subdorsal and subventral subcephalic setae (about $4 \mu \mathrm{m}$ ) and further posteriorly (about $30 \mu \mathrm{m}$ after cephalic capsule), arranged in four subdorsal and subventral groups of two or three, larger cervical setae present (15 $\mu \mathrm{m}$ long). Amphideal aperture circular and amphideal fovea pouch-shaped, small ( $8 \%$ of cbd), laterally located just posterior to capsule end. Metanemes not seen. Funnel-shaped buccal cavity with wide opening widens at level of mandibles. Its armature consists of three mandibles, each composed of two vertical rods $(16 \mu \mathrm{m})$ united by arcuate $\operatorname{bar}(7 \mu \mathrm{m})$, at top ending as left and right claws. Each mandible has tooth $(13 \mu \mathrm{m})$. The three teeth equal in size. No ocellus nor pigment spots. Pharynx cylindrical with irregular contours, cardia not visible. Nerve ring lying at about 33\% of pharynx length from anterior end. Excretory-secretory system not visible. Reproductive system diorchic, with opposed and outstretched testes in right position relative to intestine. One precloacal supplement, bar-shaped, located about 3 abd above cloaca. No precloacal setae present. Spicules paired, arcuate, with slight manubrium (1.4 abd). Gubernaculum small (26\% of spicule, $0.4 \mathrm{abd}$ ), embracing spicule, two dorso-caudal apophyses present. The gubernaculum divided into two parts. Proximal part with two small pointed teeth, surrounds tips of spicules. Arched dense zone, shaped like ring, separates it from distal triangular part. Tail 3.4 abd long, conical with end part cylindrical ( $1 / 5$ approximately). Few short caudal setae can be seen. Caudal gland bodies in pre-anal region. Terminal setae not present. Cuticle around spinneret hardly englobed. 


\section{Female}

Similar to males in general body shape, anterior sensilla, amphideal fovea and cuticle. Females longer and larger than males. Lips not rounded but pointed. Short somatic setae present all along body $(9-12 \mu \mathrm{m})$ in greater quantity than in males. Reproductive system didelphic amphidelphic, with two antidromously reflexed ovaries, positioned left of intestine. Vulva and vagina conspicuous, with associated musculature. Vulva not sclerotized. Tail conical, ending in $1 / 5$ cylindrical part, but this part less differentiated than in males. Caudal gland bodies in pre-anal region. Many short caudal setae. Terminal setae not present. Cuticle around spinneret englobed.

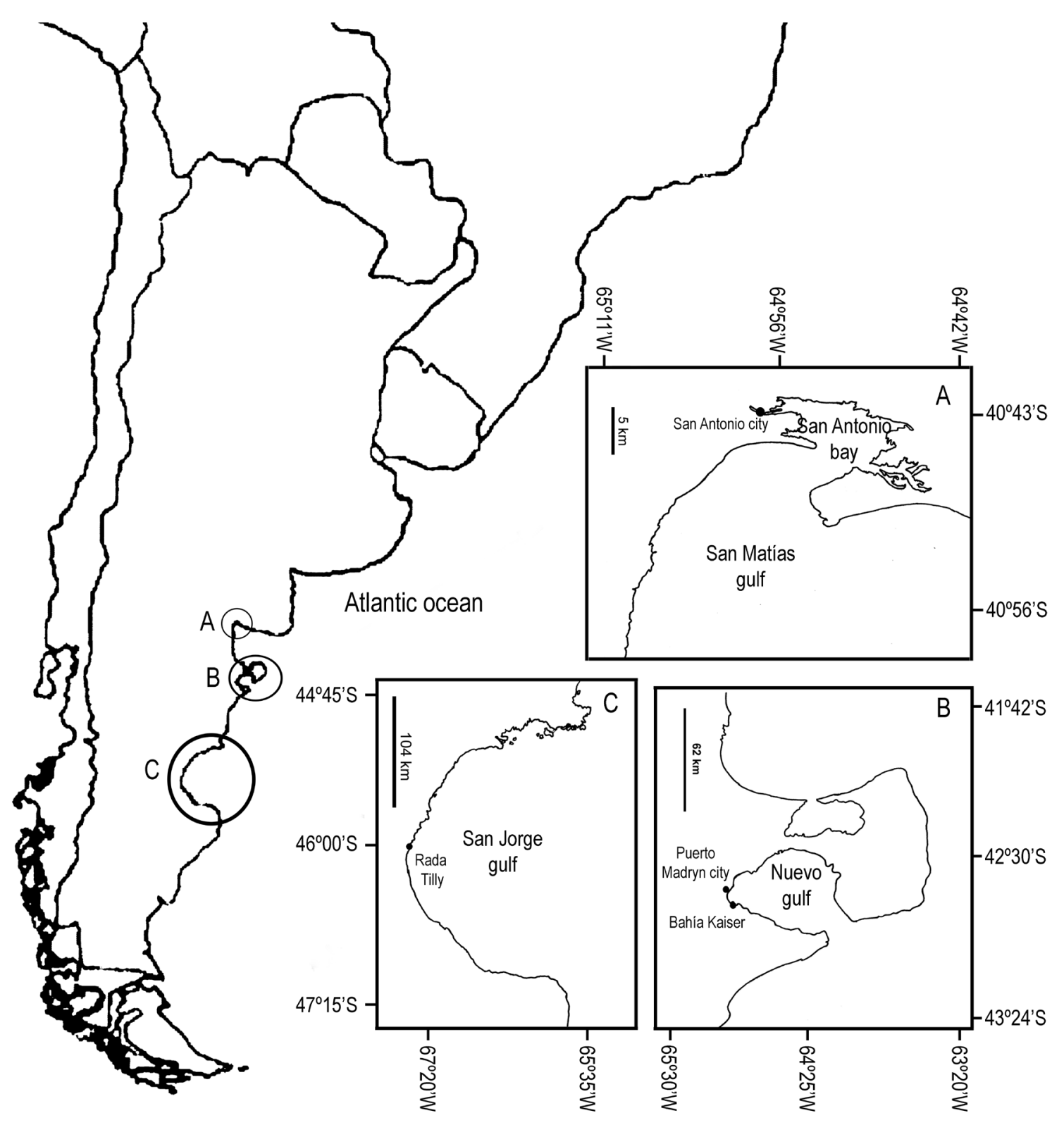

Fig. 1. Map showing the study sites; map of sampling areas. A. 'San Antonio Oeste river estuary', Río Negro Province. B. 'Puerto Madryn city' and 'Bahía Kaiser', Chubut Province. C. 'Rada Tilly', Chubut Province. 

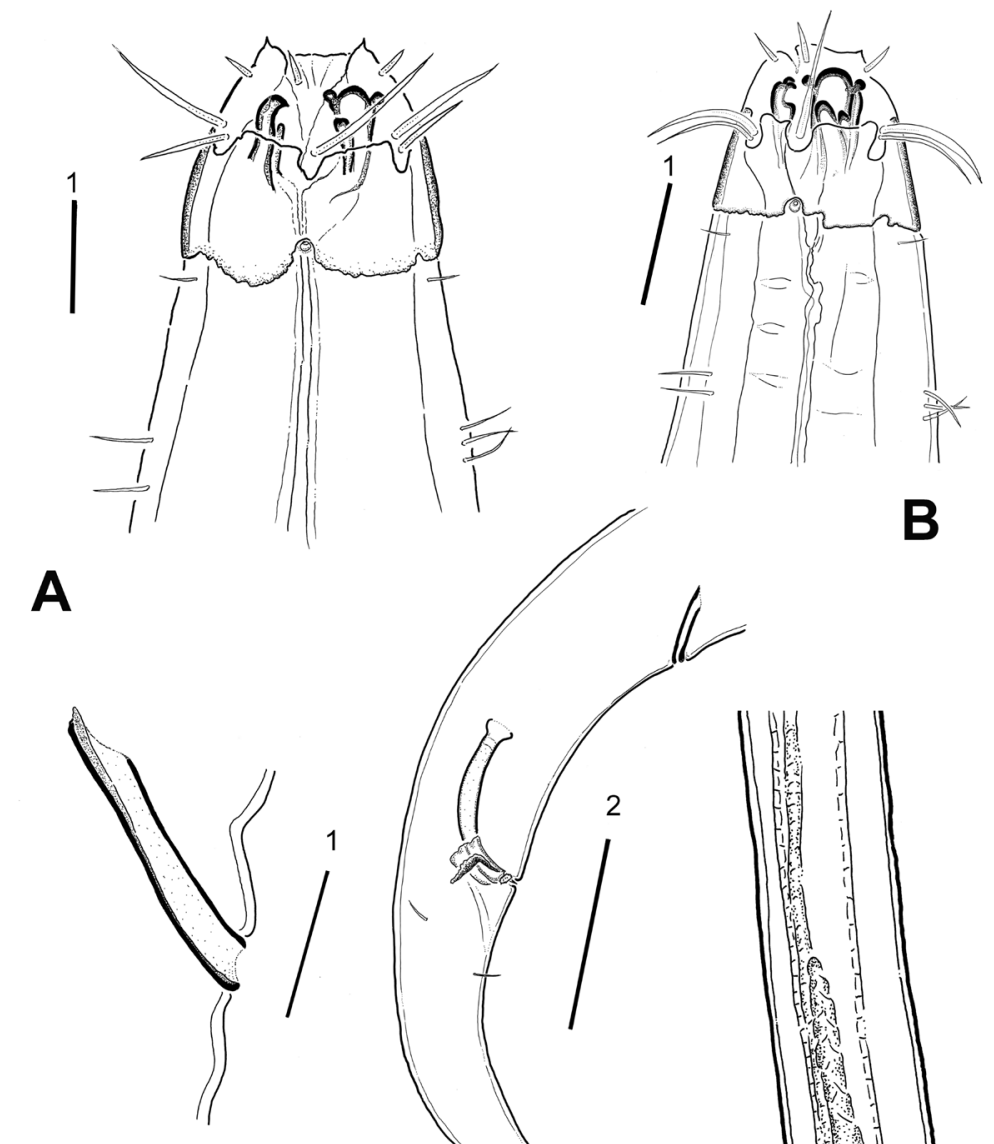

D
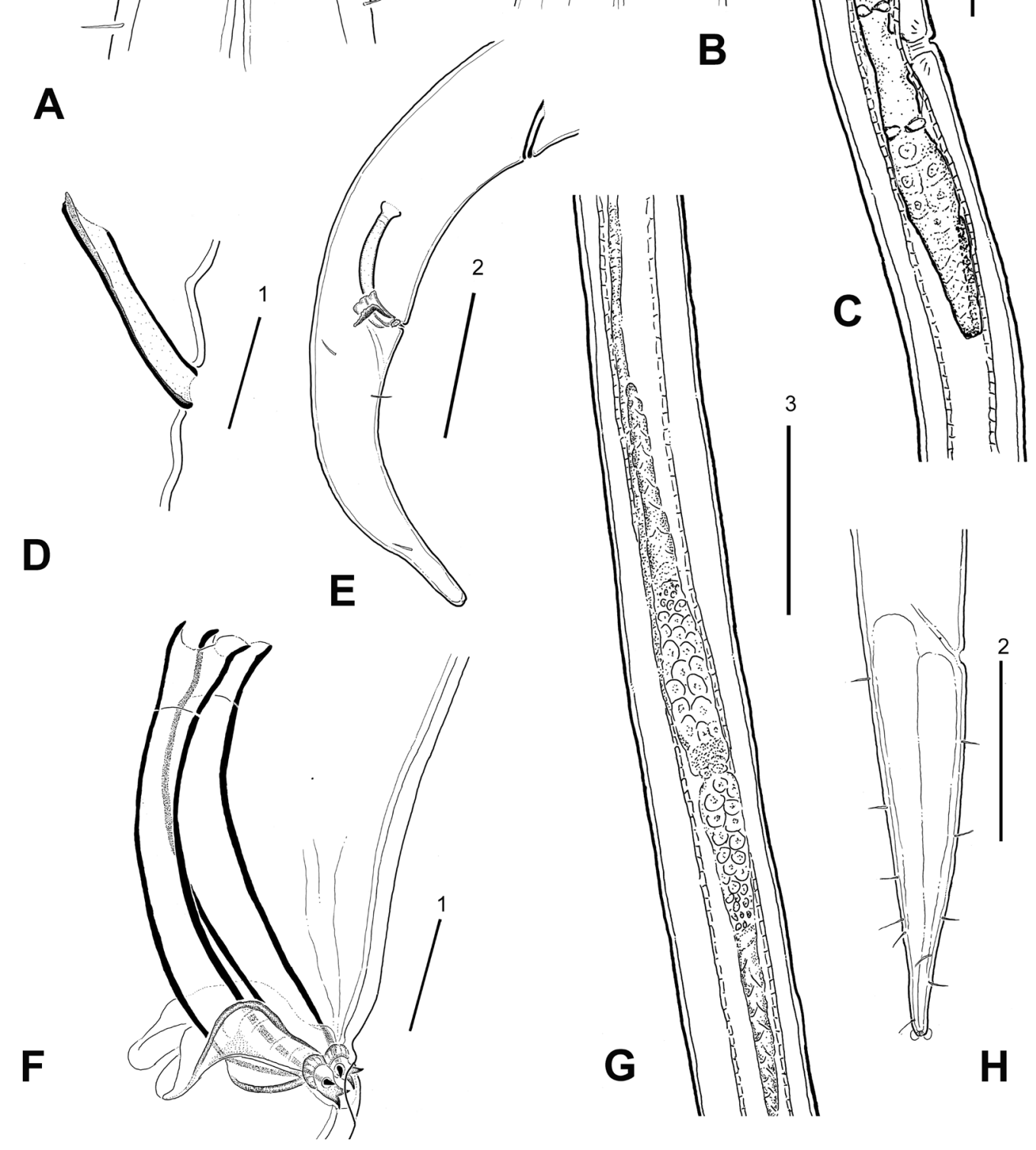

B

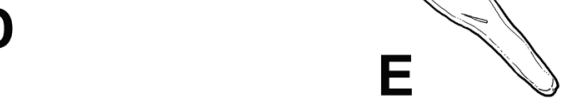

Fig. 2. Mesacanthion bifidum sp. nov. A. Cephalic sense organs on anterior end of female paratype (CNPNEM 983). B. Cephalic sense organs on male holotype (CNP-NEM 936). C. Female paratype (CNPNEM 983) showing vulva and gonadal apparatus. D. Precloacal supplement organ of male holotype (CNP-NEM 936). E. Posterior end of male holotype (CNP-NEM 936). F. Copulatory apparatus, spicules and gubernaculum of male holotype (CNP-NEM 936). G. Gonads of male holotype (CNP-NEM 936). H. Posterior end of female paratype (CNP-NEM 983). Scale bars: $1=20 \mu \mathrm{m} ; 2=100 \mu \mathrm{m} ; 3=200 \mu \mathrm{m}$. 


\section{Differential diagnosis}

Mesacanthion bifidum sp. nov. is characterized by its smooth cuticle, relatively short labial and cephalic setae, onchia of equal size, amphidial fovea pouch-shaped, spicule arcuate, gubernaculum with pointed tips at the proximal end and dorso-caudal apophysis, and tail conical-cylindrical without terminal setae.

Following the key of Jeong et al. (2019), our new species is closely related to M. virile. Both species share some characteristics, such as spicules length less than 2 abd and gubernaculum with dorso-caudal apophysis and triangular shape with two parts. The male in M. bifidum sp. nov. has the outer labial setae less than 1 cephalic diameter in length, whereas in M. virile they are about 1.25 cephalic diameters. The position of the precloacal supplementary organ in $M$. virile is closer to the cloaca $(1.1$ spicule length distant from the anus) than in M. bifidum sp. nov. (2.7 spicule lengths distant from the anus) and the spicules of both species are quite different. M. virile has an L-shaped spicule with a mid-projection for muscle insertion, whereas M. bifidum sp. nov. has an arcuate spicule without projections. On the other hand, the gubernaculum also has differences in shape. Although in both species the distal part is rather similar, the proximal part is rod-like in M. virile, whereas in M. bifidum sp. nov. two-pointed structures surround the spicule tip in the posterior position.

\section{Mesacanthion longigubernaculum sp. nov. urn:1sid:zoobank.org:act:CBD8B1B5-E4C4-44A4-8B45-3180296C8AC3}

Figs 3, 5A, C

\section{Etymology}

In reference to the length of the gubernaculum.

\section{Material examined}

\section{Holotype}

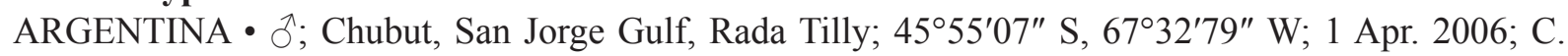
Pastor leg.; high littoral; fine sand sediments; CNP-NEM 27442.

\section{Paratypes}

ARGENTINA・2 ふえ; same collection data as for holotype; CNP-NEM 27259 and 27443.

\section{Description}

\section{Measurements}

See Table 1.

\section{Male (holotype)}

Long and slender body. Cuticle slightly striated. Cephalic region set off with presence of delicate cephalic capsule ( $7 \mu \mathrm{m}$ in height). Cephalic capsule with same thickness throughout its smooth surface. Anterior edge located above level of cephalic setae. Posterior end of cephalic capsule almost straight. No ocellus nor pigment spots. Three rounded lips, higher than mandibles. Each lip carries two inner labial setae $(8.5 \mu \mathrm{m}$ long). Six outer labial setae (18 $\mu \mathrm{m}$ long), longer than four cephalic setae (13 $\mu \mathrm{m}$ long), located at half the total height of cephalic capsule. Posterior to cephalic capsule one crown of subcephalic setae formed by six pairs of setae with one short $(7 \mu \mathrm{m})$ and one long seta $(20 \mu \mathrm{m})$. Then, crown of cervical setae ( $20 \mu \mathrm{m}$ behind first) formed by four setae, two in subdorsal position and two in subventral position, and eight setae $(7-20 \mu \mathrm{m})$ in same position as first crown. After nerve ring, another crown composed of groups of 4-5 short and scattered setae (about $4 \mu \mathrm{m}$ ) arranged in rows beside one longer seta $(20 \mu \mathrm{m})$. Rest of body presents few long somatic setae (about $14 \mu \mathrm{m})$ until cloaca. Amphideal aperture lentil-shaped, small (14\% of cbd) located just posterior to capsule end. Metanemes not seen. 


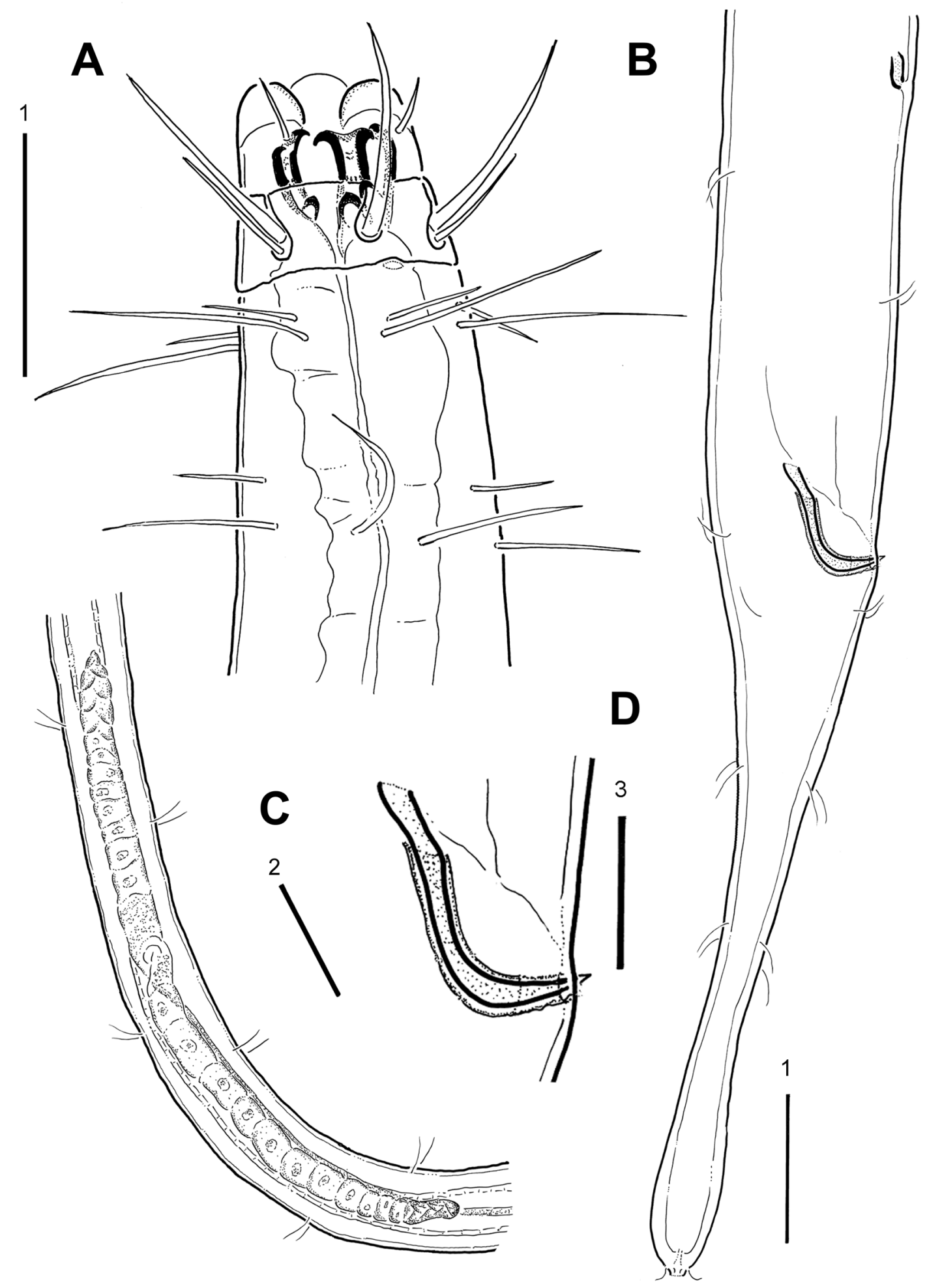

Fig. 3. Mesacanthion longigubernaculum sp. nov., $\widehat{\jmath}$, holotype (CNP-NEM 27442). A. Cephalic sense organs on anterior end. B. Posterior end, showing copulatory apparatus and precloacal supplement organ. C. Gonads. D. Copulatory apparatus, spicules and gubernaculum. Scale bars: $1=20 \mu \mathrm{m} ; 2=$ $100 \mu \mathrm{m} ; 3=10 \mu \mathrm{m}$. 
Funnel-shaped buccal cavity with wide opening diminishing its width towards level of mandibles to their base. Mandibles composed of two vertical rods $(10 \mu \mathrm{m})$ united by delicate arcuate bar $(4 \mu \mathrm{m})$ at top and ending as claws. Dorsal tooth slightly smaller than two ventrosublateral teeth. All teeth pointed. Pharynx cylindrical with irregular contours. Cardia triangular and going into intestine. Nerve ring lying at about $36 \%$ of pharynx length from anterior end. Secretory-excretory system not visible. Reproductive system diorchic, with opposed and outstretched testes in right position relative to intestine. One small precloacal bar-shaped supplement located about 3 abd above copulatory organ, almost parallel to body wall. Subventral precloacal setae present. Spicules paired, with distal portion arcuate $(2 / 3)$ and proximal portion straight and dorsally directed (1/3). Gubernaculum without apophysis, barely evident surrounding spicule, $63 \%$ of spicule. Tail 4.6 abd long, conical in first part ( $(1 / 3)$ with end part cylindrical $(2 / 3$ approximately). Caudal gland bodies in pre-anal region. Few caudal setae of same length as somatic setae and two small $(3 \mu \mathrm{m})$ terminal setae present.

\section{Female}

Unknown.

\section{Differential diagnosis}

Mesacanthion longigubernaculum sp. nov. is characterized by its long and slender body, striated cuticle, relatively long cephalic and cervical setae, onchia of different sizes, amphidial fovea lentil-shaped, spicule arcuate, gubernaculum surrounding the spicule, and tail conical-cylindrical with terminal setae.

Following the key of Jeong et al. (2019), M. longigubernaculum sp. nov. is similar to M. longissimesetosum and M. pali. However, M. longigubernaculum sp. nov. differs from M. longissimesetosum in having shorter inner and outer labial setae ( 8 and 19 vs 12 and 65-70 $\mu \mathrm{m}$, respectively) and cephalic setae (12 vs $65 \mu \mathrm{m}$ ), a higher $a$ index ( $52.9 \mathrm{vs} 30$ ), and a different shape of the gubernaculum. Mesacanthion pali has longer inner and outer labial setae ( 24 and 84 vs 8 and $19 \mu \mathrm{m}$, respectively), lower $b$ and $c^{\prime}$ indices $(b=3$ vs $4.5 ; c^{\prime}=2.8$ vs 4.4$)$, a longer cephalic capsule $(26 \mathrm{vs} 16 \mu \mathrm{m})$, a gubernaculum with well-developed apophysis and the precloacal organ located at more than 2 spicule lengths from the anus.

Mesacanthion sanantoniensis sp. nov. urn:1sid:zoobank.org:act:79D5AC77-6294-4397-A91C-8DC83E8F02DB

Figs 4, 5B, E

\section{Etymology}

Named after the locality where this species was found.

\section{Material examined}

\section{Holotype}

ARGENTINA • đ’; Río Negro, San Matías Gulf, San Antonio Oeste; 4043’ S, 6458’ W; 10 Oct. 2006; C. Pastor leg.; lower littoral; fine sand sediments; CNP-NEM 849.

\section{Paratypes}

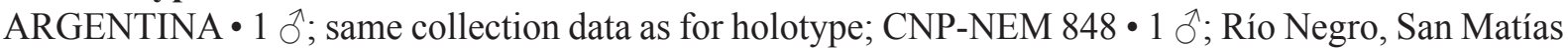
Gulf, San Antonio Oeste; 40 $43^{\prime}$ S, 64 $56^{\prime}$ W; 17 Jun. 2006; C. Pastor leg.; lower littoral; fine sand sediments; MACN-In 43853.

\section{Description}

\section{Measurements}

See Table 1. 


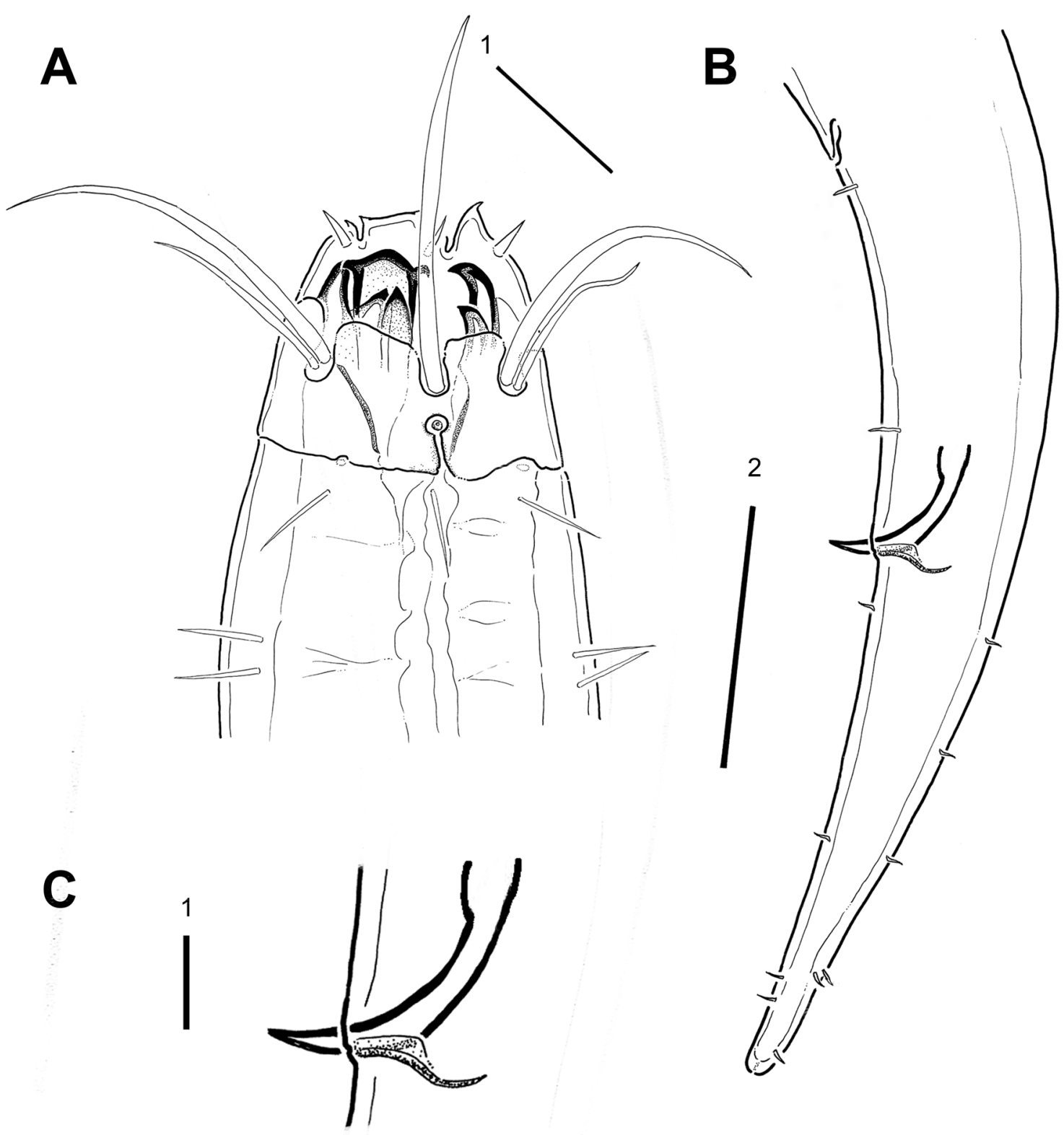

D

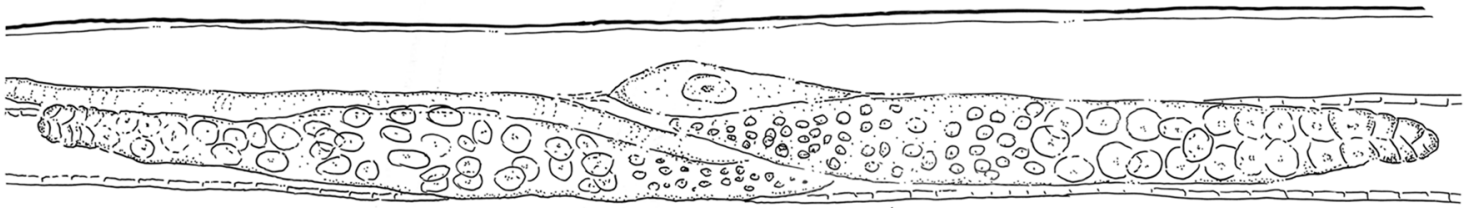

Fig. 4. Mesacanthion sanantoniensis sp. nov., $\widehat{\jmath}$, holotype (CNP-NEM 849). A. Cephalic sense organs on anterior end. B. Posterior end, showing copulatory apparatus and precloacal supplement organ. C. Copulatory apparatus, spicules and gubernaculum. D. Gonads. Scale bars: $1=20 \mu \mathrm{m} ; 2=100 \mu \mathrm{m}$. 

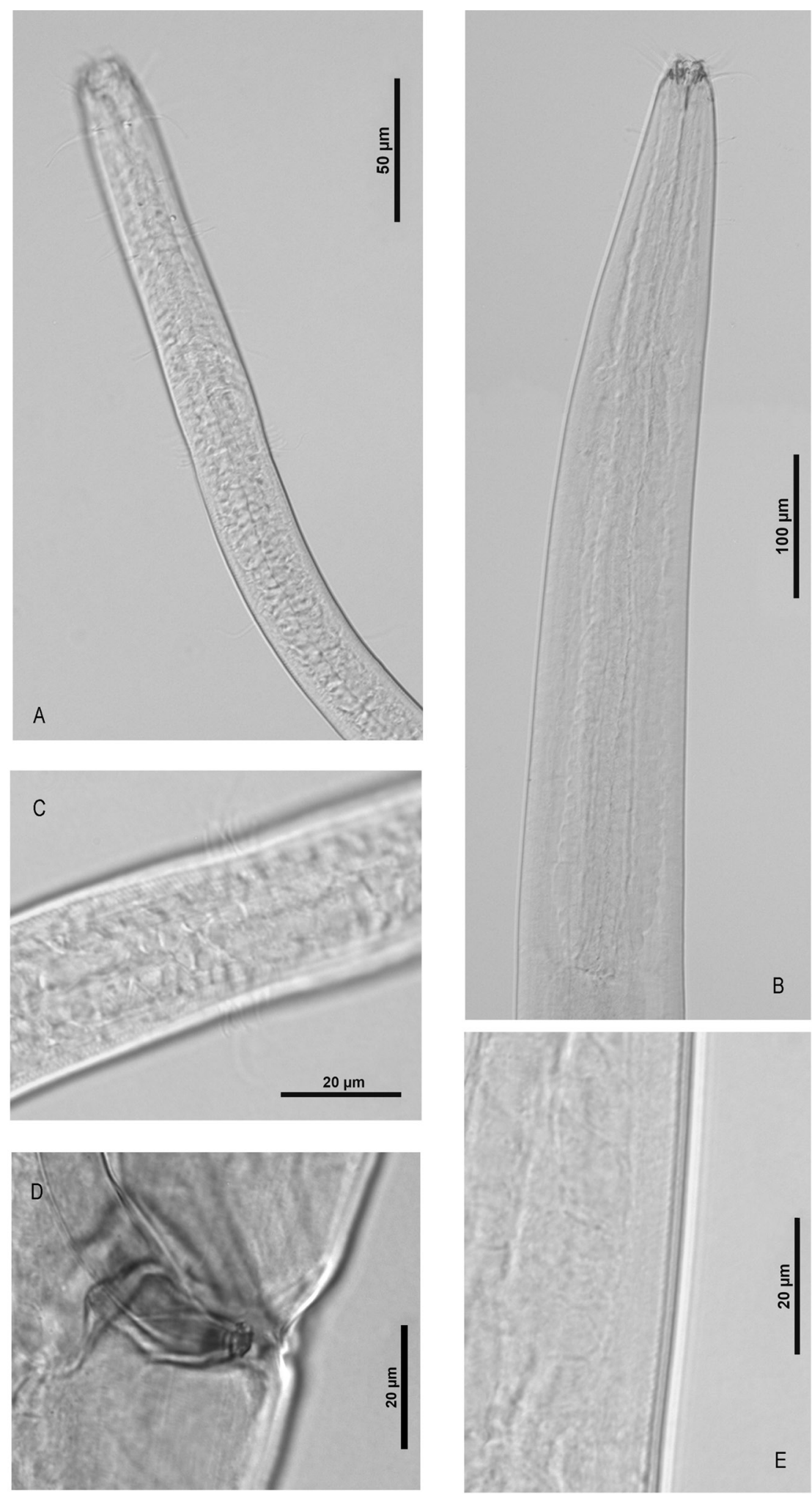

Fig. 5. A. Pharyngeal region of Mesacanthion longigubernaculum sp. nov., $\widehat{\jmath}$, holotype (CNP-NEM 27442). B. Pharyngeal region of $M$. sanantoniensis sp. nov., ô, paratype (CNP-NEM 848). C. Detail of M. longigubernaculum sp. nov., §̂, holotype (CNP-NEM 27442), showing cuticular striation. D. Gubernaculum of M. bifidum sp. nov., ô, holotype (CNP-NEM 936). E. Detail of M. sanantoniensis sp. nov., ${ }^{\Uparrow}$, paratype (CNP-NEM 848), showing cuticular striation. 
Male (holotype)

Large and stout body. Cuticle slightly striated. Cephalic region set off with presence of cephalic capsule (16 $\mu \mathrm{m}$ in height). Cephalic capsule with same thickness throughout its rough surface. Anterior edge located above level of cephalic setae. Posterior end of cephalic capsule almost straight. No ocellus nor pigment spots. Three high lips with points facing outward. Each lip carries two inner labial setae ( $7 \mu \mathrm{m}$ long). Six outer labial setae ( $32 \mu \mathrm{m}$ long) double length of four cephalic setae (16 $\mu \mathrm{m}$ long), located at middle of cephalic capsule arranged in single crown. Near and posterior to cephalic capsule, short subcephalic setae (about 8-9 $\mu \mathrm{m}$ ), two in subventral position, two lateral and two in subdorsal position. About $25 \mu \mathrm{m}$ after cephalic capsule, toward nerve ring, pairs of cervical setae in subventral and subdorsal positions (same size as previous). Same size setae present between nerve ring and end of oesophagus, but not in pairs and scattered. Rest of body presents few shorter somatic setae (about $4 \mu \mathrm{m}$ ) until cloaca, except for longer one $(8 \mu \mathrm{m})$ located just before precloacal organ. Amphideal aperture pore-shaped, small ( $7 \%$ of cbd), located at middle of cephalic capsule. Metanemes not seen. Funnel-shaped buccal cavity with wide opening that diminishes its width towards level of mandibles to their base. Its armature consists of three mandibles composed of two vertical rods $(15 \mu \mathrm{m})$ united by arcuate bar $(9 \mu \mathrm{m})$ at top and ending as claws. Each mandible associated with tooth forming a unit. Dorsal tooth slightly smaller $(9 \mu \mathrm{m})$ than two ventrosublateral teeth $(11 \mu \mathrm{m})$. Tip of teeth not very pointed. Pharynx cylindrical with irregular contours. Cardia barely visible, triangular and going into intestine. Nerve ring lying at about $33 \%$ of pharynx length from anterior end. Secretory-excretory system not visible. Reproductive system diorchic, with opposed and outstretched testes in right position relative to intestine. One ventral precloacal bar-shaped supplement located about 2.4 abd anterior to cloaca. Subventral precloacal setae present. Spicule paired, arcuate, with slight seam forming manubrium (1.5 abd). Gubernaculum with dorsal apophysis ( $29 \%$ of spicule), perpendicular to body axis. Tail 4.1 abd long, conical. It presents few setae in subventral and subdorsal positions $(8 \mu \mathrm{m})$. Caudal gland bodies in anal region. Terminal setae not present.

\section{Female}

Unknown.

\section{Differential diagnosis}

Mesacanthion sanantoniensis sp. nov. is characterized by its long and stout body, striated cuticle, long cephalic setae, onchia of different sizes, amphidial fovea pouch-shaped, spicule arcuate, gubernaculum with dorsal apophysis, and tail conical without terminal setae.

As $M$. longigubernaculum sp. nov., M. sanantoniensis sp. nov. is similar to $M$. longissimesetosum and M. pali, according to the key of Jeong et al. (2019). They have males with a supplementary organ lying further away from the cloaca, presence of gubernaculum, and presence of subventral precloacal setae. Mesacanthion sanantoniensis sp. nov. has shorter inner and outer labial setae than M. pali (7.3 and 33 vs 24 and $92 \mu \mathrm{m}$, respectively), shorter cephalic setae (20.2 vs $84 \mu \mathrm{m})$, lower $a$ index (24.7 vs 50), and higher $b$ (4.1 vs 3.3) and $c^{\prime}$ (4.1 vs 2.8) indices, shorter mandibles (15 vs $\left.25 \mu \mathrm{m}\right)$ and a smaller precloacal organ $(19.3$ vs $31 \mu \mathrm{m})$. Mesacanthion longissimesetosum and $M$. sanantoniensis sp. nov. have differences in mandible shape. Mesacanthion sanantoniensis sp. nov. has mandibles ending in jaws, posteriorly straight and slightly smaller. Mesacanthion longissimesetosum has mandibles posteriorly arcuate and larger. Mesacanthion sanantoniensis sp. nov. has a distinct amphid and three crowns of cervical setae. Mesacanthion longissimesetosum has no amphid, and the cervical setae are not arranged in crowns. Mesacanthion longissimesetosum has two tiny precloacal setae just before the spicule vs none in $M$. sanantoniensis sp. nov. At the posterior part of the tail, the new species has two pairs of setae plus one single seta, and M. longissimesetosum has none. 
Considering the similarities between $M$. longigubernaculum sp. nov., M. sanantoniensis sp. nov., M. longissimesetosum and M. pali, we provide a small key for an easy differentiation.

\section{Key to species of Mesacanthion with simple spicules shorter than 2 abd and subventral precloacal setae present}

This key can be taken as an addition of the last entrance (22) in the key to species of Mesacanthion in Jeong et al. (2019) to include two new species.

1. Precloacal supplement organ less than 2 spicule lengths distant to cloaca ...... M. pali Wieser, 1959

- Precloacal supplement organ more than 2 spicule lengths distant to cloaca

2. Gubernaculum without dorsal apophysis

M. longigubernaculum sp.nov.

- Gubernaculum with dorsal apophysis 3

3. De Man's $b$ index greater than 5

M. longissimesetosum Wieser, 1953

- De Man's $b$ index smaller than 5 M. sanantoniensis sp. nov.

\section{Discussion}

With the new species described in this study, the total number of Mesacanthion species amounts to 42 . For South America, the number of species present is 11 including new and previously known species. The present work contributes with three new species to the general knowledge of the taxonomy of freeliving marine nematodes and the genus of Mesacanthion, in particular.

Species of Mesacanthion are distributed all around the world, mostly in fine sand and mud sediments. Each one of the new species in this study was found in a different gulf on the Patagonian coasts. This results in an expansion in the distribution of the genus to new areas. They were found at different horizontal littoral and sublittoral zones, but all were inhabiting fine sand. This is also relevant for the biodiversity and ecology information of South America and Argentina.

\section{Acknowledgments}

This work was partly supported by GEF-PNUD Project 02/018 A-B-55. The authors thank the two anonymous reviewers, whose recommendations improved the present work.

\section{References}

Bezerra T.N., Eisendle-Flöckner U., Hodda M., Holovachov O., Leduc D., Mokievsky V., Peña Santiago R., Sharma J., Smol N., Tchesunov A., Venekey V., Zhao Z. \& Vanreusel A. 2021. Nemys: World Database of Nematodes. Available from http://nemys.ugent.be/ [accessed 30 Jun. 2021].

Cobb N.A. 1930. Marine free-living nemas. Scientific Reports of the Australasian Antartic Expedition (1911-1914). Ser. C. Zoology \& Botany 6 (7): 1-28.

De Coninck L.A. 1965. Classe des Nématodes: Généralités. In: Grassé P.-P. (ed.) Traité de Zoologie: Anatomie, Systématique, Biologie, Tome IV, Fascicule II: Némathelminthes (Nématodes): 1-217.

De Coninck L.A. \& Schuurmans Stekhoven J.H. 1933. The freeliving marine nemas of the Belgian Coast II. With general remarks on the structure and the system of nemas. Mémoires du Musée royal d'Histoire naturelle de Belgique 58: 3-163.

De Man J.G. 1893. Cinquième note sur les nématodes libres de la Mer du Nord et de la Manche. Mémoires de la Société zoologique de France 6: 81-125. 
Ditlevsen H. 1930. Marine free-living nematodes from New Zealand. Videnskabelige Meddelelser fra Dansk naturhistorisk Forening 87: 201-242.

Filipjev I.N. 1927. Les nématodes libres des mers septentrionales appartenant à la famille des Enoplidae. Archiv für Naturgeschichte 91 (A): 1-216.

Gerlach S.A. 1954. Nématodes marins libres des eaux souterraines littorales de Tunisie et d’Algérie. Vie et Milieu 4: 221-237.

Gerlach S.A. 1957. Die Nematodenfauna des Sandstrandes an der Küste von Mittelbrasilien (Brasilianische Meerse-Nematoden IV). Mitteilungen aus dem Zoologischen Museum in Berlin 33: 411-459. https://doi.org/10.1002/mmnz.19570330206

Gerlach S.A. \& Riemann F. 1974. The Bremerhaven Checklist of Aquatic Nematodes. A Catalogue of Nematoda Adenophorea Excluding the Dorylaimida. Part 2. Veröffentlichungen des Instituts für Meeresforschung in Bremerhaven, Bremen.

Jeong R., Tchesunov A.V. \& Lee W. 2019. Bibliographic revision of Mesacanthion Filipjev, 1927 (Nematoda: Thoracostomopsidae) with description of a new species from Jeju Island, South Korea. PeerJ 7: e8023. https://doi.org/10.7717/peerj.8023

Wieser W. 1953. Free-living marine nematodes I. Enoploidea. Acta Universitatis Lundensis, New Series 49: $1-155$.

Wieser W. 1959. Free Living Nematodes and Other Small Invertebrates of Puget Sound Beaches. University of Washington Press, Seattle.

Manuscript received: 7 July 2021

Manuscript accepted: 8 November 2021

Published on: 28 December 2021

Topic editor: Tony Robillard

Desk editor: Eva-Maria Levermann

Printed versions of all papers are also deposited in the libraries of the institutes that are members of the EJT consortium: Muséum national d'histoire naturelle, Paris, France; Meise Botanic Garden, Belgium; Royal Museum for Central Africa, Tervuren, Belgium; Royal Belgian Institute of Natural Sciences, Brussels, Belgium; Natural History Museum of Denmark, Copenhagen, Denmark; Naturalis Biodiversity Center, Leiden, the Netherlands; Museo Nacional de Ciencias Naturales-CSIC, Madrid, Spain; Real Jardín Botánico de Madrid CSIC, Spain; Zoological Research Museum Alexander Koenig, Bonn, Germany; National Museum, Prague, Czech Republic. 